\title{
QUASI-STEADY STATE COSMOLOGY
}

\author{
G. BURBIDGE \\ University of California, San Diego, 9500 Gilman Drive, La Jolla, California 92093-0111 USA \\ F. HOYLE \\ 102 Admirals Walk, Bournemouth BH2 5HF, UK \\ and \\ J.V. NARLIKAR \\ Inter-University Center for Astronomy \& Astrophysics Post Bag 4, Ganeshkhind Pune 411007, \\ India
}

\section{Introduction}

The standard big bang cosmology has the universe created out of a primeval explosion that not only created matter and radiation but also spacetime itself. The big bang event itself cannot be discussed within the framework of a physical theory but the events following it are in principle considered within the scope of science. The recent developments on the frontier between particle physics and cosmology highlight the attempts to chart the history of the very early universe.

Exciting though these studies are, they have failed to resolve some of the basic issues of cosmology. These issues can be stated briefly as follows:

1. The microwave background radiation (MBR) is considered a fundamental proof of the big bang cosmology. Yet, cosmological considerations so far have failed to deduce its present temperature of $2.73 \mathrm{~K}$.

2. While the Planckian spectrum of the MBR is deduced from its relic interpretation, its small scale anisotropy has not been successfully related to the observed large scale inhomogeneity of matter distribution in the universe, the recent findings of the satellite $C O B E$ notwithstanding.

3. There is no consistent theory of structure formation that takes in a reasonable hypothesis of dark matter and can reproduce the observed large scale structure and motions from primordial seed fluctuations.

4. The claims of explaining the large scale features of the universe in terms of discrete source populations inevitably require epicyclic hypotheses of evolution of physical properties of these sources that are post-facto rather than having any predictive power.

5. The age distribution of galaxies poses many problems for the canonical big bang model. How can we accommodate globular clusters of ages $15-18$ Gyrs in a big bang universe with $k=0$, that is required by inflation in the very early phase? Equally, it is difficult to understand the existence of very young galaxies at the present epoch, for, galaxy formation is supposed to have taken place in the early universe.

T. J.-L. Courvoisier and A. Blecha: Multi-Wavelength Continuum Emission of AGN, 293-299.

(C) 1994 IAU. Printed in the Netherlands. 
6. The phenomena in high energy astrophysics like the $Q S O s, \mathrm{AGN}$, radio sources, etc. show big outpourings of matter and energy from compact regions. However these events have no relation to the primordial big bang which is totally isolated from this relatively recent activity.

7. Finally on a theoretical issue, the big bang singularity is deduced from the equations of general relativity which are derived from an action principle. Yet, the action principle breaks down at the singularity, thus making the cycle of reasoning self-contradictory.

We believe that despite the popularity enjoyed by the big bang cosmology today, this list is sufficient to motivate an alternative approach to cosmology. (cf Arp et al. 1990, Hoyle et al. 1993 a,b).

Any alternative to big bang cosmology, should fulfill a few minimum conditions. First it must do at least as well as the big bang cosmology in explaining the MBR and light isotope abundances and in describing the observed features of discrete source surveys. Next it must try to do better than the big bang model on some of the above mentioned fronts. Finally, as a scientific theory it must make a few disaprovable predictions that distinguish it from the standard model.

In what follows we summarize a model that claims to do just that. This is the quasi steady state cosmology (QSSC) proposed by Hoyle et al. (1993 a,b).

\section{Creation of Matter}

In 1948 Bondi and Gold (1948) and Hoyle (1948) had independently proposed the steady state theory as an alternative to the big bang cosmology. Bondi and Gold had adopted the Perfect Cosmological Principle as the starting point of their approach while Hoyle had taken a field theoretic description of matter creation as the main motivation. Here we will follow the second approach but with some significant modification.

The field equations are derived from an action principle. Although Hoyle et al. (op.cit.) consider a direct particle interaction approach motivated by Mach's Principle, the following simplified derivation essentially reproduces their equations in the more familiar field theory format. Thus the classical Hilbert action leading to the Einstein equations is modified by the inclusion of a scalar field $C$ whose derivatives with respect to the spacetime coordinates $x^{i}$ are denoted by $C_{i}$. For the notation followed and further details see (Narlikar 1993). The action is given by

$$
\begin{aligned}
\mathcal{A}= & \sum_{a} \int_{\Gamma_{a}} m_{a} c d s_{a}+\int_{v} \frac{c^{3}}{16 \pi G} R \sqrt{-g} d^{4} x-\frac{1}{2 c} f \int_{v} C_{i} C^{i} \sqrt{-g} d^{4} x \\
& +\sum_{a} \int_{\Gamma_{a}} C_{i} d a^{i}
\end{aligned}
$$

where $C$ is a scalar field and $C_{i}=\partial C / \partial x^{i} . f$ is a coupling constant. The last term 
of (1) is manifestly path-independent and so, at first sight it appears to contribute no new physics. The first impression, however, turns out to be false if we admit the existence of broken worldlines.

Thus, if the worldline of particle $a$ begins at point $A$, then the variation of $\mathcal{A}$ with respect to that worldline gives

$$
m_{a} \frac{d a^{i}}{d s_{a}}=g^{i k} C_{k}
$$

at $A$. In other words, the $C$-field balances the energy-momentum of the created particle.

The field equations likewise get modified to

$$
\begin{aligned}
& R_{i k}-\frac{1}{2} g_{i k} R=-\frac{8 \pi G}{c^{4}}\left[\stackrel{T i k}{m}+{ }^{T i k} c^{i k}\right] \\
& \text { where } \stackrel{T_{c}^{i k}}{c}=-f\left\{C_{i} C_{k}-\frac{1}{2} g_{i k} C^{l} C_{l}\right\} .
\end{aligned}
$$

Thus the energy conservation law is

$$
\stackrel{T_{m}^{i k}}{m} i k=-{ }_{C}^{i k} i k=f C^{i} C^{k}{ }_{i k} .
$$

That is, matter creation via a nonzero left hand side of (5) is possible while conserving the overall energy and momentum.

The $C$-field tensor has negative stresses which lead to the expansion of spacetime, as in the case of inflation. The formalism described here is essentially that used by Hoyle and Narlikar $(1962,1966 \mathrm{a}, \mathrm{b})$ in the 1960s to produce inflation type solution (which, of course, predated Guth's inflationary cosmology by 15 years!).

From (2) we therefore get a necessary condition for creation as

$$
C_{i} C^{i}=m_{a}^{2} c^{4}
$$

This is the 'creation threshold' which must be crossed for particle creation. How this can happen near a massive object can be seen from the following simple example.

The Schwarzschild solution for a massive object $M$ of radius $R>2 G M / c^{2}$ is

$$
d s^{2}=c^{2} d t^{2}\left(1-\frac{2 G M}{c^{2} r}\right)-\frac{d r^{2}}{1-\frac{2 G M}{c^{2}} r}-r^{2}\left(d \theta^{2}+\sin ^{2} \theta d \phi^{2}\right)
$$


for $r \geq R$. Now if the $C$-field does not seriously change the geometry, we would have at $r \gg R, \dot{C} \approx \alpha$ and $C^{\prime} \approx 0$, so that

$$
C^{i} C_{i} \equiv\left(1-\frac{2 G M}{c^{2} r}\right)^{-1} \frac{\alpha^{2}}{c^{2}}
$$

In other words $C_{i} C^{i}$ increases towards the object and can become arbitrarily large if $R \approx 2 G M / c^{2}$. So it is possible for the creation threshold to be reached near a massive collapsed object even if $C_{i} C^{i}$ is below the threshold far away from the object. In this way massive collapsed objects can provide new sites for matter creation. Thus, instead of a single big bang event of creation, we have mini-creation events (MCEs) near collapsed massive objects.

\section{A Cosmological Solution}

Since the $C$-field is a global cosmological field, we expect the creation phenomenon to be globally cophased. Thus, there will be phases when the creation activity is large, leading to the generation of the $C$-field strength in large quantities. However, the $C$-field growth because of its large negative stresses leads to a rapid expansion of the universe and a consequent drop in its background strength. When that happens creation is reduced and takes place only near the most collapsed massive objects thus leading to a drop in the intensity of the $C$-field. The reduction in $C$ field slows down the expansion, even leading to local contraction and so to build-up of the $C$-field strength. And so on!

We can describe this up and down type of activity as an oscillatory solution superposed on a steadily expanding de Sitter type solution of the field equations by a scale factor that varies with cosmic time $t$ as follows:

$$
S(t)=\exp \left(\frac{t}{P}\right)\left\{1+\alpha \cos \frac{2 \pi t}{Q}\right\} \text {. }
$$

Note that the universe has a long term secular expanding trend, but because $|\alpha|<$ 1 , it also executes non-singular oscillations around it. We can determine $\alpha$ and our present epoch $t=t_{0}$ by the observations of the present state of the universe. Thus an acceptable set of parameters is $\alpha=0.75, t_{0}=0.85 Q, Q=4 \times 10^{10} y r ., P=20 Q$. Although the set is not unique and there will be a range of acceptable values, we will work with this set to illustrate the performance of the model.

\section{The Origin of Nuclei and the Microwave Background}

We have as yet not said what particle is being created by the $C$-field. The answer is, the Planck particle whose mass is

$$
m_{p}=\sqrt{\frac{3 \hbar}{4 \pi G}} \sim 10^{-5} g
$$


This particle, however, has a very short lifetime $\sim 10^{-44} s$. It decays ultimately into the baryon octet and radiation. Most members of the octet except $n$ and $p$ are also short-lived and decay into protons. Only the neutron and the proton combine into stable helium nuclei. Thus approximately $25 \%$ by mass (2 out of 8 baryons) combine to form helium.

A more careful calculation gives the helium mass fraction to be around $23 \%$, with a tiny fraction of $1-2 \%$ in the form of metals. This type of nucleosynthesis also generates ${ }^{2} \mathrm{H},{ }^{3} \mathrm{H},{ }^{3} \mathrm{He},{ }^{6} \mathrm{Li},{ }^{7} \mathrm{Li},{ }^{9} \mathrm{Be},{ }^{10} \mathrm{Be}$ etc. in small amounts that are in agreement with the observations, see Appendix I in Hoyle et al. (1993 a). In fact, the abundances of the light isotopes in this cosmology lead to a better agreement with observations than in the big bang model.

There is one further important consequence. In the big bang model the required production of deuterium imposes a stringent upper limit on the present day baryon density. This limit forces the conventional big-bang cosmologists to asume that the dark matter component of the universe must be largely nonbaryonic. In the QSSC, there is no such density limit from deuterium abundance and thus the dark matter component can be baryonic.

What about the microwave background? The $Q S S C$ obtains it in the following way. First, each Planck particle decay is like a fireball: it produces lots of energy, including baryons $\left(\sim 10^{19}\right.$ per Planck particle) and radiation. More than the hot big bang, the Planck fireball can provide several interesting and realistic studies in astroparticle physics. Further, since the Planck fireballs are repeated phenomena, rather than the 'once only' type situation of the hot big bang cosmology, they are amenable to more exhaustive scientific study.

The bulk of the fireball energy goes into expansion. However, some radiation remains as a relic of the fireball. Together with the starlight generated in the preceding oscillatory cycles this energy is to be thermalized to provide the microwave background. Does it provide enough radiant energy to give a $2.7 \mathrm{~K}$ background? Is the background thoroughly thermalized to produce a black body spectrum? Also, is it homogeneous to the extend given by $C O B E$ (Smoot, et al 1992) and other measurements? Quantitative studies (see Hoyle, et al. 1993 a,b) answer all these questions in the affirmative.

The starlight from several past generations of stars is sufficient to maintain a steady background of radiation whose present temperature is calculated to be $\sim 2.7 K$, provided, some agency is available to thermalize it. The agency proposed is dust in the form of metallic needles, mostly of iron, which absorb the ambient radiation and reradiate it in the microwave region. Provided this has gone on long enough, the radiation spectrum will hae an accurate black body form. Calculation shows that indeed the thermalization has occurred through as many as $10^{3}$ absorptions and re-emissions by iron whiskers - sufficient to ensure an extremely close approximation to the black body curve. The iron whiskers are typically $\sim 1 \mathrm{~mm}$ 
in length and $10^{-6} \mathrm{~cm}$ in radius of cross section. The iron itself is produced partly from stellar nucleosynthesis in supernovae and partly fromt he decay of the Planck particle. The required density in the form of such whiskers is only $\sim 10^{-35} \mathrm{~g} \mathrm{~cm}^{-3}$ : well within the observed cosmic abundances of iron.

The background produced will be very smooth with a patchiness of density of the order of $10^{-5}$. Fluctuations of density and temperature of this or larger order get smoothed out by redistribution of iron grains by the radiation pressure. On smaller scales the dynamical smoothness-restoring forces are too small to make the radiation smooth. Thus, the $C O B E$ finding of $\triangle T / T \sim 10^{-5}$ is consistent with the above picture. Moreover, the characteristic scale of $10^{26} \mathrm{~cm}$ at the oscillatory minimum will expand to $\sim 5 \times 10^{26} \mathrm{~cm}$ at present, giving a characteristic angular scale for the above patichiness to be $\sim 10^{\circ}$, in conformity with the $C O B E$ scale of angular inhomogeneity.

In our papers (Hoyle et al. 1993 a,b) we have discussed the redshift-apparent magnitude relation, the counts of radio sources and the angular diameter redshift relation all with in the framework of the QSSC. Because of the shortage of space, and the subject matter discussed at this meeting, we now turn directly to the cosmogony associated with the QSSC.

\section{The Cosmogony Associated with the QSSC}

(i) Violent Events in Galaxies What we see all about us is a violent universe in which from a variety of centers large amounts of energy in the form of photons and particles are being ejected. In the radio galaxies and in the QSOs and in the nuclei of many galaxies we know that we are seeing the results of non-thermal energy production often arising through the incoherent synchrotron mechanism. Also in Seyfert nuclei and the like we see the ejection of large amounts of kinetic energy in the form of hot gas moving at velocities $\sim 10^{9} \mathrm{~cm} \mathrm{sec}^{-1}$. There is also extensive circumstantial evidence based on geometrical configurations and optical morphology suggesting that coherent objects are ejected from the nuclei of galaxies. Finally the $\gamma$-ray burst sources, if they lie at cosmological distances may very well be direct evidence for creation.

This whole class of phenomena is in our view best interpreted as direct evidence for matter creation on a small scale - minicreation events (MCE) as was originally proposed by Ambartsumian $(1958,1965)$ and Jeans (1929).

Other phenomena which we believe can best be explained by the QSSC include

(ii) The Age of the Universe According to QSSC the universe is infinitely old but the average age of astronomical objects is $1 / 3 P \sim 3 \times 10^{11} \mathrm{yrs}$. This makes many clusters much older than hitherto assumed. Even our Galaxy might have age of this order with several generations of stars formed, evolved and burnt 
out. The dark matter component in the Galaxy may be largely made of burnt out stars.

(iii) Hierarchy of Structures The largest structure to form in the MCEs is the so called supercluster of mass $\sim 10^{15}--10^{16} M_{\odot}$. There are, however, MCEs on smaller scales going right down to galactic nuclei with masses $\sim 10^{6}-10^{7} M_{\odot}$. It is, however, the former that keep the universe going steady state at all times.

Finally there is the possibility that gravity wave sources may be due to creation events. Narlikar and Das Gupta (1993) have shown that such events in the mass range $100-1000 M_{\odot}$ can be detected by the laser interferometric detectors being planned. Further the gravity wave background created by such MCEs may also affect the timing mechanism of millisecond pulsars by an amount that is detectable.

\section{References}

Ambartsumian, V.A., $(1958,1965)$ Proc. of the Solvay Conf. on the Structure of the Universe 241, and, Proc. of the Solvay Conf. on The Structure and Evolution of the Galaxies 1.

Arp, H.C., Burbidge, G., Hoyle, F., Narlikar, J.V. and Wickramasinghe, N.C. (1990) Nature, 346, 807.

Bondi, H. and Gold, T. (1948) M.N.R.A.S., 108, 252.

Hoyle, F. (1948) M.N.R.A.S., 108, 372.

Hoyle, F. and Narlikar, J.V. (1962) Proc. Roy. Soc. A270, 334.

Hoyle, F. and Narlikar, J.V. (1966) Proc. Roy. Soc. A290, 143.

Hoyle, F. and Narlikar, J.V. (1966) Proc. Roy. Soc. A290, 162.

Hoyle, F., Burbidge, G. and Narlikar, J.V. (1993 a) Ap.J., 410, 437.

Hoyle, F., Burbidge, G. and Narlikar, J.V. (1993 b), preprint.

Jeans, J. (1929) Astronomy \& Cosmogony (Cambridge Univ. Press) 352.

Narlikar, J.V. (1993) Introduction to Cosmology, 2nd Edition, (Cambridge Univiversity Press).

Narlikar, J.V. and Das Gupta, P. (1993) M.N.R.A.S., to be published.

Smoot, G.F., et al., (1992) Ap.J., 396, L1. 